\title{
Calculation of persistent currents in superconducting magnets
}

\author{
C. Völlinger, M. Aleksa, and S. Russenschuck \\ CERN, 1211 Geneva 23, Switzerland
}

(Received 27 September 2000; published 13 December 2000)

\begin{abstract}
This paper describes a semianalytical hysteresis model for hard superconductors. The model is based on the critical state model considering the dependency of the critical current density on the varying local field in the superconducting filaments. By combining this hysteresis model with numerical field computation methods, it is possible to calculate the persistent current multipole errors in the magnet taking local saturation effects in the magnetic iron parts into consideration. As an application of the method, the use of soft magnetic iron sheets (coil protection sheets mounted between the coils and the collars) for partial compensation of the multipole errors during the ramping of the magnets is investigated.
\end{abstract}

PACS numbers: 41.20.Gz, 74.20.De

\section{INTRODUCTION}

The Large Hadron Collider (LHC) [1], a proton-proton superconducting accelerator, will consist of about 8400 superconducting magnet units of different types, all operating in superfluid helium at a temperature of $1.9 \mathrm{~K}$. Field variations in the LHC superconducting magnets, e.g., during the ramping of the magnets, induce magnetization currents in the superconducting filaments. These so-called persistent currents do not decay but persist due to the lack of resistivity. The resulting multipole field errors in the magnets are small in the case of nominal current but non-negligible at injection field level, where the injected proton beam has a large emittance.

The persistent current distribution has been modeled by means of the critical state model [2], taking into account their dependency on the magnetic induction by use of a current fit [3] (since the critical currents cannot be measured directly). Single filaments have been discretized into elliptically shaped layers with varying current density according to the fit. In the LHC main dipole magnet, which is used as an example, the local field in the coil varies depending on the excitation current and changing yoke saturation during the ramping of the magnets. Therefore, the computation of the filament magnetization takes into account the local position in the coil as well as their hysteretic behavior.

The magnetization model is combined with the coupled boundary element/finite element method (BEM-FEM method) [4] for the computation of the local field in the coil. The BEM-FEM formulation has the major advantage that the representation of the coil in the finite element mesh can be avoided since the coil is positioned in the iron-free BEM domain. The fields arising from current sources in the coil (including the persistent currents) are calculated analytically by means of the Biot-Savart law. Hence, the discretization errors due to the finite-element part in the BEM-FEM formulation are limited to the iron magnetization arising from the surrounding yoke structure, which accounts for about $20 \%$ of the total field. The method also avoids artificial (far field) boundary conditions. The BEM-FEM coupling is specially suited for the computation of thin layers of different materials which can be independently meshed as they are connected through the boundary element domain (see also Sec. III B).

Together with an $M(B)$-iteration scheme for the filament magnetization, the method was used for the computation of a thin ferromagnetic coil protection sheet. With a sheet of suitable thickness the nonlinearities in the multipole errors during the upramp cycle of the dipoles can be significantly reduced.

\section{SUPERCONDUCTING FILAMENT MAGNETIZATION}

The LHC main dipole magnet is wound of a keystoned Rutherford type cable, containing 28 and 36 strands on the inner and outer coil layers, respectively. The strands of the cable are made of NbTi filaments of 6 and $7 \mu \mathrm{m}$ in diameter (inner and outer layer of the coil) and are embedded in a copper matrix. According to the critical state model of Bean [2], a hard superconductor tries to expel any external field by generating a bipolar screening current distribution of critical density $\pm J_{c}$.

The critical state model is originally derived for a slab of superconducting material and has been modified for cylindrical filaments by Wilson [5]. The dependency of the critical current density $J_{c}$ on the magnetic induction is taken into account by a current fit [3], and the discretization of the filaments in elliptic layers, as shown in Fig. 1.

This semianalytical approach permits one to use a current fit that shows a very good agreement with measurements both for low and high external magnetic fields. However, integration over this current density fit cannot be done analytically, since no closed solution has been found.

Current distributions in filaments can be modeled as elliptically shaped [5], since the applied dipole field in the cross section is perpendicular to the filament axis but not necessarily fully penetrating. The model allows each layer to flip individually in case the external field changes 


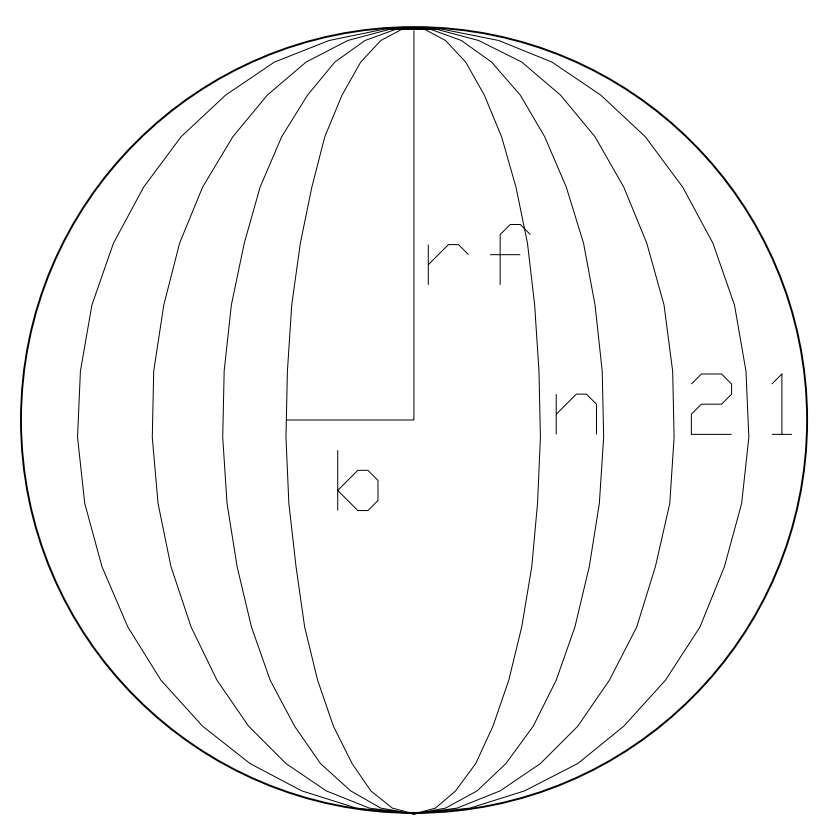

FIG. 1. Discretized filament with elliptically layered current distribution.

orientation and hence accounts for local field variations in the coil arising from yoke saturations during the ramping of the magnets.

The screening field and the magnetization of one elliptical current layer is given by Eqs. (1) in the case of circular outer shape. In a case of noncircular outer shape, the current layers have to be superposed. Here, the magnetization is defined as the magnetic moment per unit volume, caused by the induced screening currents,

$$
\begin{aligned}
B_{\text {screen }} & =\frac{2 \mu_{0} J_{c} r_{f}}{\pi}\left(1-\sqrt{1-\varepsilon^{2}} \frac{\arcsin (\varepsilon)}{\varepsilon}\right) \text { in }[\mathrm{T}], \\
M & =\frac{4}{3 \pi} r_{f} J_{c} \varepsilon^{2} \text { in }[\mathrm{A} / \mathrm{m}] .
\end{aligned}
$$

The parameter $\varepsilon$ represents the ellipticity and $r_{f}$ the filament radius. In case the local external field exceeds the maximum screenable value of the filament (fully penetrated state), the magnetization takes its peak value where $\varepsilon$ equals 1 .

\section{HYSTERESIS MODEL AND NUMERICAL FIELD COMPUTATION}

\section{A. Field harmonics}

The magnetic field components in the aperture of superconducting magnets are commonly given in so-called multipoles in the two-dimensional case. Multipoles are the coefficients of the Fourier series expansion of the field at a certain reference radius in the aperture of a magnet, obtained after harmonic analysis. From the radial component of the magnetic flux density $B_{r}$ as a function of the angular position $\varphi$ at a given reference radius $r=r_{0}$, one gets for the Fourier-series expansion of the field

$$
\begin{aligned}
B_{r}^{\infty} & =\sum_{n=1}^{\infty}\left(B_{n} \sin n \varphi+A_{n} \cos n \varphi\right) \\
& =B_{N} \sum_{n=1}^{\infty}\left(b_{n} \sin n \varphi+a_{n} \cos n \varphi\right) .
\end{aligned}
$$

The field components are related to the main field component $B_{N}$ in order to express the relative multipoles $b_{n}$, where $N=1$ for a dipole, $N=2$ for a quadrupole, etc. The $B_{n}$ are denoted as the normal and the $A_{n}$ the skew components of the field given in $\mathrm{T}, b_{n}$ are the normal relative and $a_{n}$ the skew relative field components (multipoles). They are dimensionless and given in units of $10^{-4}$ at a $17 \mathrm{~mm}$ reference radius.

\section{B. The BEM-FEM coupling method}

The general field problem for the BEM-FEM computation is shown in Fig. 2. As can be seen in the figure, the BEM-FEM computing area comprises different domains: Inside the nonmeshed air domain (boundary element domain) $\Omega_{3}$, the superconducting coil is positioned. Additional magnetic subdomains of different material

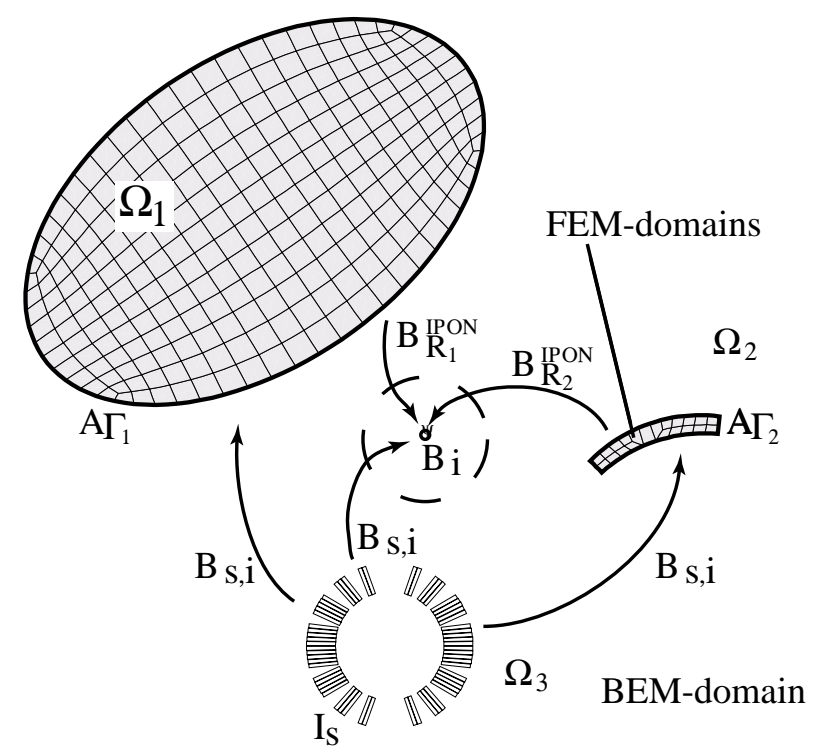

FIG. 2. General field problem for BEM-FEM computation of superconducting magnets. The BEM-FEM area comprises the air domain $\Omega_{3}$ where the superconducting coil and the evaluation point are positioned. The source currents in the coil $I_{\mathrm{S}}$ act on the different magnetic subdomains $\Omega_{1}$ and $\Omega_{2}$ (partly nonlinear). In the case of the computation of superconducting magnets, the evaluation point is inside the aperture of the magnet in the air domain (symbolized by the dashed circle). The magnetic field in the evaluation point comprises contributions of the arbitrarily shaped nonlinear iron yoke (here, subdomain $\Omega_{1}$ ), the thin soft magnetic iron sheet (here, subdomain $\Omega_{2}$ ), and the superconducting source currents. 
parameters $\left(\Omega_{1}\right.$ and $\left.\Omega_{2}\right)$, discretized in finite elements, exist. The magnetic subdomains can be arbitrarily shaped and nonlinear magnetic material parameters are allowed. The evaluation point is positioned in the BEM domain (free from magnetic material and source currents), symbolized by the dashed circle. Only the magnetic domains have to be meshed with finite elements.

The field arising from the superconducting coil can be computed analytically by means of the Biot-Savart law, since the coil is positioned in the air subdomain $\Omega_{3}$ of the BEM-FEM area. Single currents in the coil are accurately described by single line currents at the position of the superconducting strands. From the source currents, one gets the source vector potential $A_{\Gamma_{S}}$ on the coupling boundary between the FEM domains and the air domain. One gets the resulting vector potentials $A_{\Gamma_{1}}$ and $A_{\Gamma_{2}}$ from the iterative solution of the system of linear equations that results from the BEM-FEM coupling method. In this case, the right-hand side of the system of equations is the vector potential $A_{\Gamma_{S}}$, which can again be calculated by Biot-Savart-type integrals [6]. The reduced magnetic inductions $\mathbf{B}_{\mathbf{R}_{1}}$ and $\mathbf{B}_{\mathbf{R}_{2}}$ can be computed by means of Kirchhoff integrations, once the $A_{\Gamma}$ are determined. One gets the magnetic induction at the evaluation point by superposing the source field $\mathbf{B}_{\mathbf{S}}$ and the iron contributions $\mathbf{B}_{\mathbf{R}_{\mathrm{n}}}$.

In the case of the LHC main dipoles, about $20 \%$ of the total field in the aperture is contributed by the magnetization of the iron yoke structure. Since the BEM-FEM coupling method computes the source fields analytically, the numerical errors are restricted to the numerically computed $20 \%$ of the field arising from the iron yoke.

\section{The $M(B)$ iteration}

The scheme of the $M(B)$ iteration and the feedback of the superconducting filament magnetization are explained by means of a flow chart.

The parameters shown in the flow chart for the computation of persistent currents are as follows: $I_{\mathrm{S}}$ presents the total current (source current) driven during one LHC cycle while $I_{\mathrm{n}}$ is the individual transport current in single strands. $\mathbf{B}_{\mathbf{S}, \mathbf{i}}$ is the source field at the $i$ th strand position and is calculated from the Biot-Savart law. $A_{\Gamma}$ is the $z$ component of the vector potential on the BEM-FEM coupling boundary used to compute $\mathbf{M}^{\mathbf{I R O N}}$, which is the magnetization arising from the surrounding ferromagnetic (iron) yoke. The magnetization is defined as the magnetic moment per unit volume. $\mathbf{B}_{\mathbf{R}, \mathbf{i}}^{\mathbf{I R O N}}$ represents the reduced magnetic induction due to iron magnetization and is computed by solving Kirchhoff integrals. Hence, one gets the magnetic induction at the $i$ th strand position $\mathbf{B}_{\mathbf{i}}$ by superposing the source field and the reduced field arising from iron magnetization.

The induced bipolar persistent currents are computed from the superconducting filament magnetization. They are added to the source currents and iterations with updated source fields are performed until convergence is obtained. As can be seen in the flow chart (Fig. 3), the parameter $\mathbf{M}_{\mathbf{i}}^{\text {PERS }}$ represents the superconducting filament magnetization which results from the magnetic induction at the $i$ th strand position (the external field seen by the superconductor). $\mathbf{B}_{\mathbf{i}}$ itself is the sum of the magnetic induction resulting from the filamentary currents, being obtained by means of the Biot-Savart law, and the reduced magnetic induction due to iron magnetization. The reduced magnetic induction is computed by solving Kirchhoff integrals. $A_{\Gamma}$ denotes the vector potential on the BEM-FEM coupling boundary, which is in the first step calculated from the source currents only, but in the second step takes into account the contribution from persistent currents as well. The

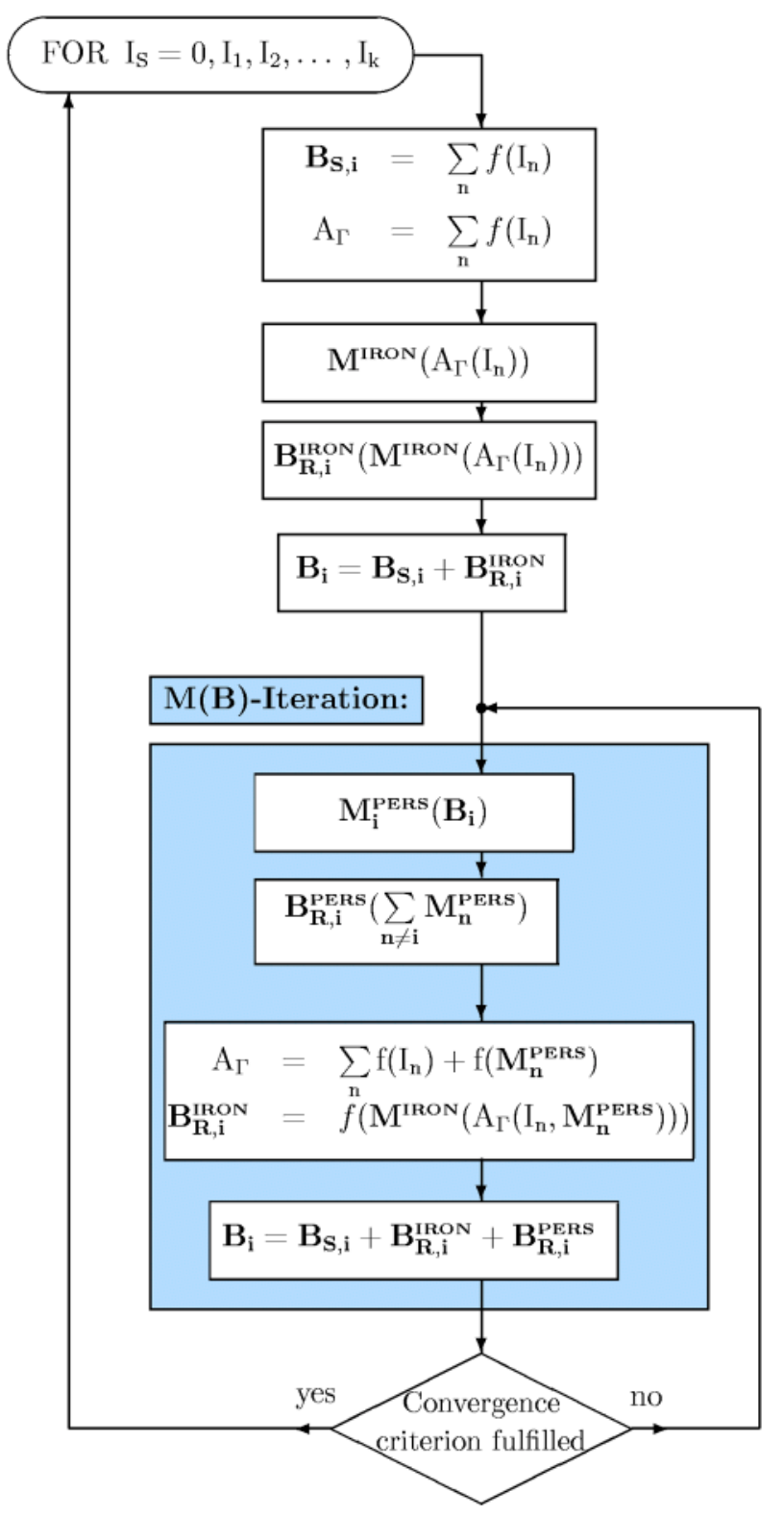

FIG. 3. (Color) Algorithm for the computation of persistent currents in ROXIE 8.0. 
total magnetic induction $\mathbf{B}_{\mathbf{i}}$ at the $i$ th strand position results from superposition of the source field, the persistent current, and the iron contribution. The BEM-FEM method used is described in detail in [4].

\section{MAGNETIZATION IN THE LHC DIPOLE COIL}

The external field seen by individual filaments depends on their position in the coil geometry. Filaments in the outer layer of the coil (close to abscissa) experience low fields [dark blue regions in Fig. 4(b)], but high field variations when the eddies of the field move outwards during upramping. Filaments in the inner coil layer experience higher field [red and purple regions in Fig. 4(b)], which is increasing with inverse dependency to the positioning angle [see Fig. 4(b)]. The modulus of the superconducting filament magnetization in the coil cross section is shown in Fig. 4(a). It is observed that even at nominal field there are
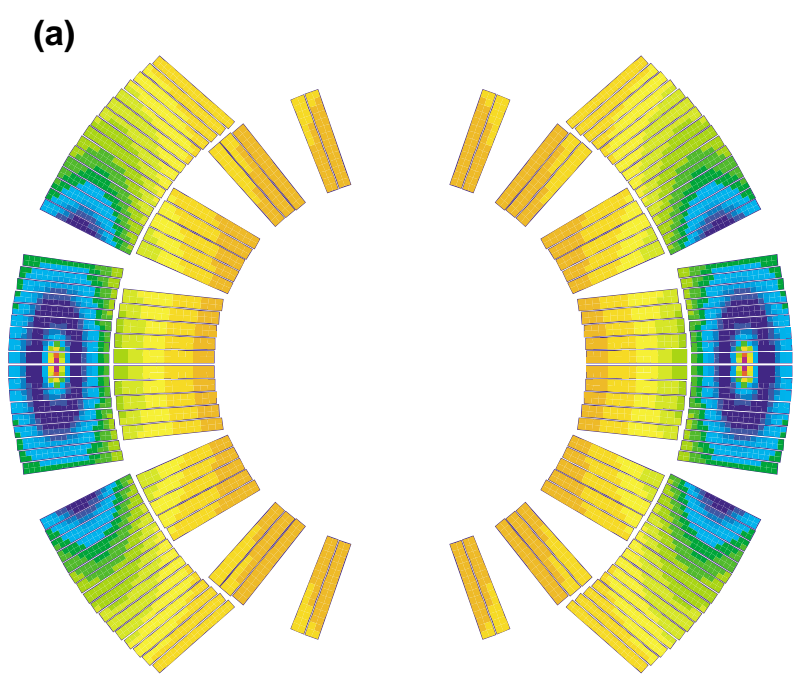

(b)

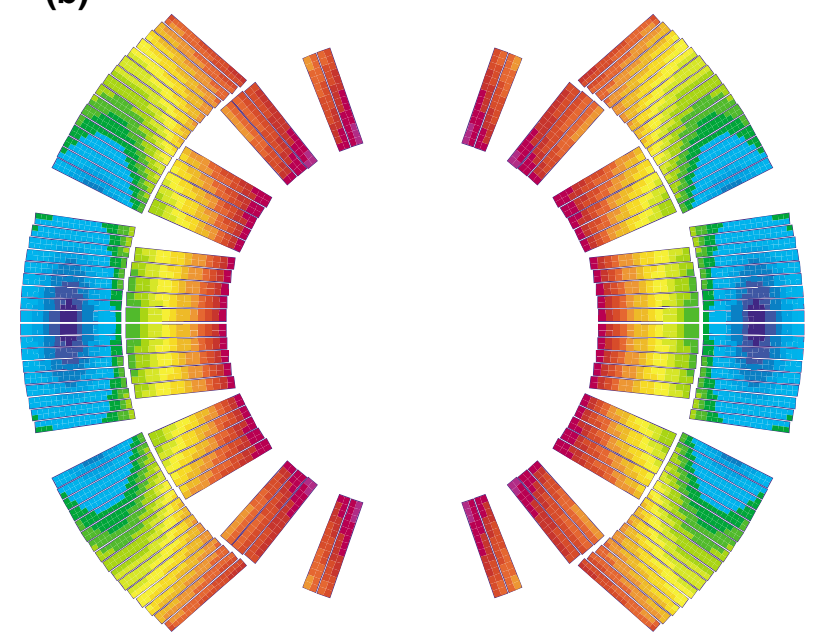

FIG. 4. (Color) (a) Modulus of superconducting filament magnetization and (b) modulus of magnetic field in the coil, both at injection field level.

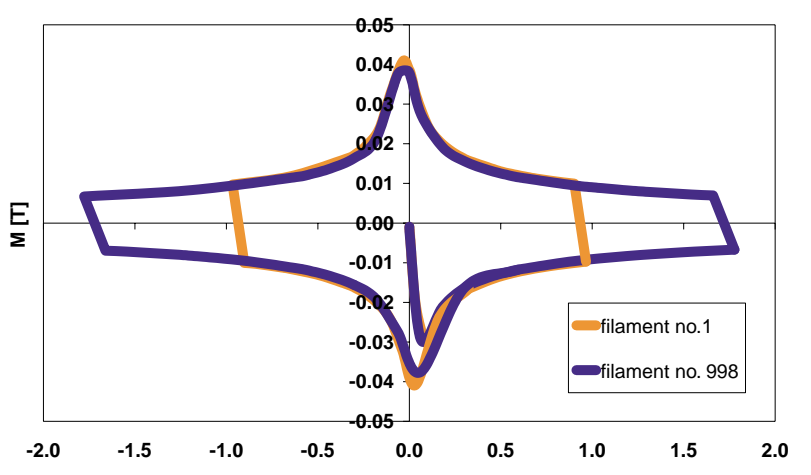

FIG. 5. (Color) Computed magnetization curves of two superconducting filaments at different positions in the coil cross section. Blue curve: outer coil layer, close to the abscissa; red curve: inner coil layer, angle approximately $50^{\circ}$ with respect to the abscissa.

filaments in the coil cross section still remaining nonfully penetrated.

A complete up-down-ramp cycle for two filaments on different positions in the coil cross section is shown in Fig. 5 in order to demonstrate the hysteretic behavior. The curve also shows that the shift of the magnetization curve with respect to the ordinate axis (hysteretic effect due to the persistence of induced currents while passing the null value of the external field) arises without being explicitly modeled.

\section{PART COMPENSATION OF PERSISTENT CURRENT INDUCED MULTIPOLE ERRORS BY MEANS OF SOFT MAGNETIC IRON SHEETS}

Different approaches for the computation of field distortions due to persistent currents have been discussed in literature, as well as the passive compensation for induced persistent currents; Brück et al. [7] calculated field errors arising from persistent currents while the compensation has been done mostly in the form of passive superconducting correction strips inside the aperture of the coil [8] or on the wedges between the coil blocks [9]. Green [10] also partly compensated persistent current effects by placing ferromagnetic pieces within the bore of the magnet or on the coil wedges, again.

Here, an intrinsic solution for the compensation of the persistent current multipole errors of the LHC dipoles has been found by making the so-called coil protection sheets (CPS) from soft magnetic material of $0.5 \mathrm{~mm}$ thickness. The coil protection sheets are placed on the outer coil radius and the material properties assumed are those of iron sheets with a very low content of impurities (99.99\% pure $\mathrm{Fe}$ ).

The ferromagnetic sheet caused the nonlinearities in the upramp cycle on the $b_{3}$ multipole component to be reduced by a factor of 4 (while decreasing the $b_{5}$ variation by a factor of 2). Figure 6 shows the geometric model of the soft magnetic coil protection sheet for the twin aperture 


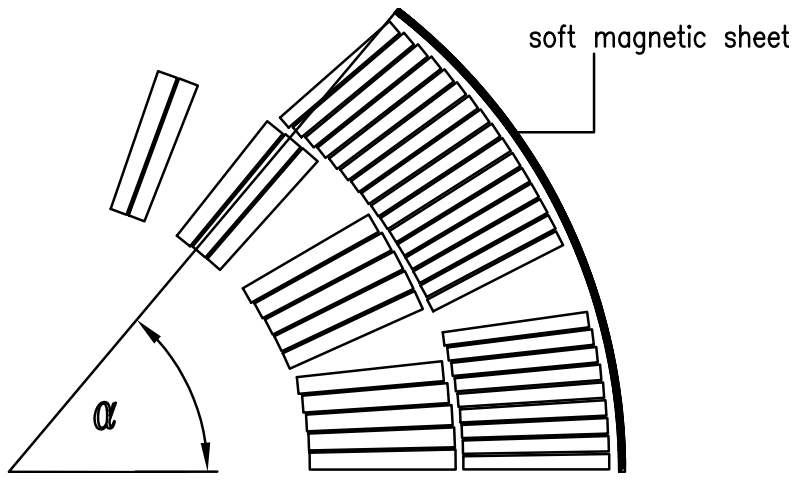

FIG. 6. First quadrant of LHC dipole coil with the soft magnetic coil protection sheet.

dipole. The sheet is mounted between the outer coil radius and the collars. The first quadrant of the complete finite element geometry as used for the computation is shown in Fig. 7.

Additionally, using sheets of slightly different thicknesses offers a tuning possibility for the series magnet coils and can compensate deviations [11] arising from cables of different suppliers. The computed field distribution in the dipole magnet is shown in Fig. 8.

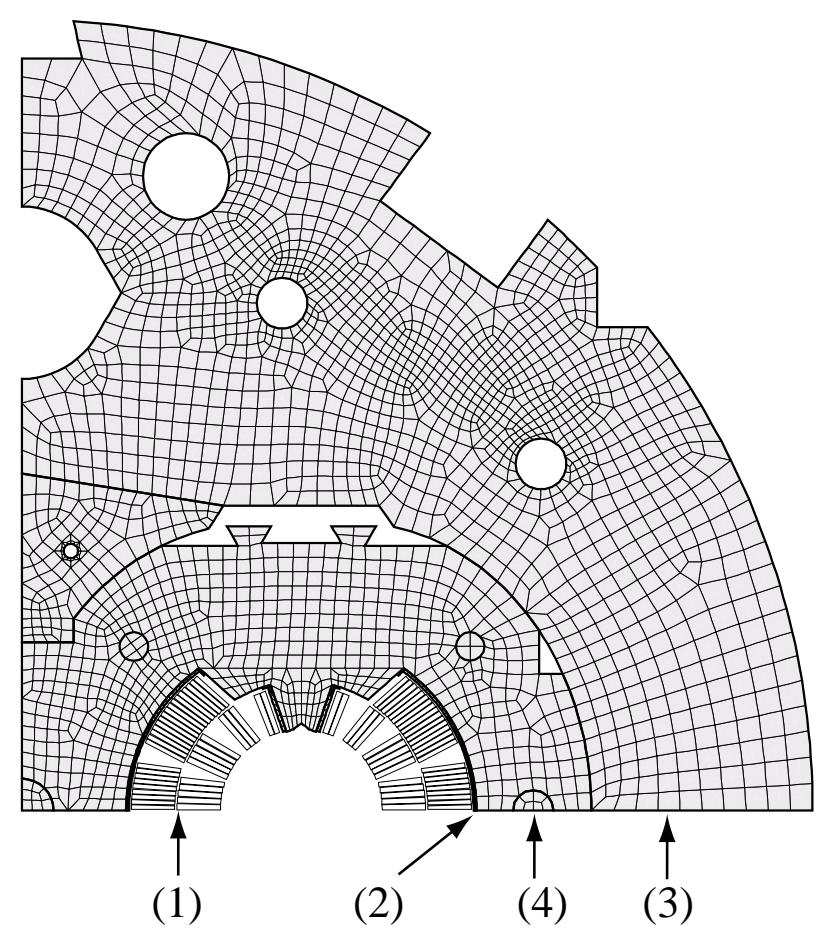

FIG. 7. Finite element geometry comprising the nonlinear iron yoke (3), the stainless steel collars (4), the partly ferromagnetic coil protection sheet (2), and the superconducting coil (1). As can be seen, the coil protection sheet consists of two pieces, partly covering the inner layer and completely covering the outer layer of the coil. Only the circular shaped piece of the outer coil layer is made of ferromagnetic material as shown in Fig. 6, while all other parts are made of nonmagnetic stainless steel.
Table I shows the computed multipole field errors at injection and nominal field level in the LHC main dipole for a coil protection sheet made completely from nonmagnetic and partly from soft magnetic material, respectively.

The computation has been done in the cross section with a relative permeability $\mu_{r}=1$ of the stainless steel collars (collars shown, e.g., in Fig. 7). The lower order multipole errors as a function of the excitation are displayed in Fig. 9 (all values are in units of $10^{-4}$ at $17 \mathrm{~mm}$ reference radius).

Table I and the excitation curves (Fig. 9) show that the soft magnetic coil protection sheet reduces the variation of all lower order multipoles. The main improvements are the reduction of the nonlinearities in the upramp cycle on the $b_{3}$ multipole component by a factor of 4 while decreasing the $b_{5}$ variation by a factor of 2 . The main objective in using the ferromagnetic coil protection sheet is the reduction of the multipole variation during the ramping of the magnets since any biasing of the curves can easily be reduced by a slight shift of a coil block in the cross section. After the design optimization of the six-block coil in 1998, changes in the coil geometry and the yoke were undertaken that resulted in an overcompensation of the persistent currents in the present design. The results presented above now allow a readjustment of the coil layout of the series magnets in such a way that the offset of the multipole curves vanishes while keeping the multipole variation on the very low level presented in Fig. 9 (continuous curves).

The compensation of persistent currents by means of making the coil protection sheet from ferromagnetic material is restricted by the contradictory influence on the multipoles $b_{3}$ and $b_{5}$. Hence, two independent parameters were taken for the optimization: variation of the thickness, which has been found optimal at $0.5 \mathrm{~mm}$, and the angle up to which the coil is covered with ferromagnetic material (indicated as $\alpha$ in Fig. 6).

Figure 10 shows the dependency of the multipoles on the angle $\alpha$. The curves show that an optimal angle of $46.34^{\circ}$ with respect to the abscissa exists that has been found after

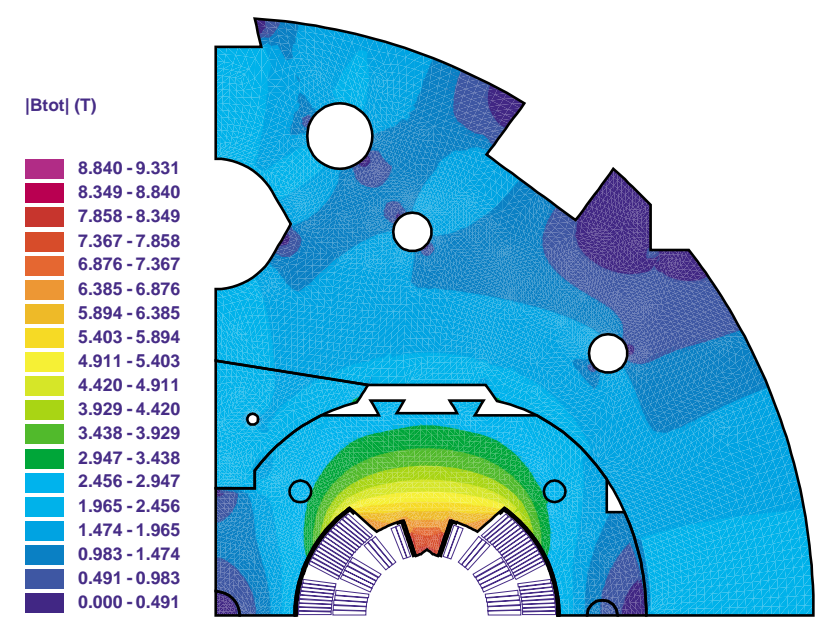

FIG. 8. (Color) Modulus of the magnetic induction in the first quadrant of the yoke cross section for the LHC dipole geometry. 
TABLE I. Expected relative multipole errors including persistent current contribution in units of $10^{-4}$ at 17 mm reference radius.

\begin{tabular}{crrrrrr}
\hline \hline & \multicolumn{2}{c}{$b_{n}$ at injection field } & \multicolumn{2}{c}{$b_{n}$ at nominal field } & \multicolumn{2}{c}{$\Delta b_{n}$ (upramp) } \\
Soft magnetic CPS & \multicolumn{1}{c}{ No } & \multicolumn{1}{c}{ Yes } & \multicolumn{1}{c}{ No } & \multicolumn{1}{c}{ Yes } & No \\
\hline$b_{2}$ & -3.063 & -2.937 & -3.193 & -3.157 & 0.935 & 0.914 \\
$b_{3}$ & -1.376 & 6.957 & 6.759 & 8.293 & 8.135 & 2.344 \\
$b_{4}$ & 0.080 & 0.080 & -0.013 & -0.012 & 0.114 & 0.111 \\
$b_{5}$ & -0.131 & -1.288 & -0.911 & -0.959 & 0.779 & 0.328 \\
$b_{6}$ & 0.004 & 0.002 & -0.001 & -0.001 & 0.005 & 0.005 \\
$b_{7}$ & 0.242 & 0.241 & 0.629 & 0.633 & 0.387 & 0.392 \\
\hline \hline
\end{tabular}
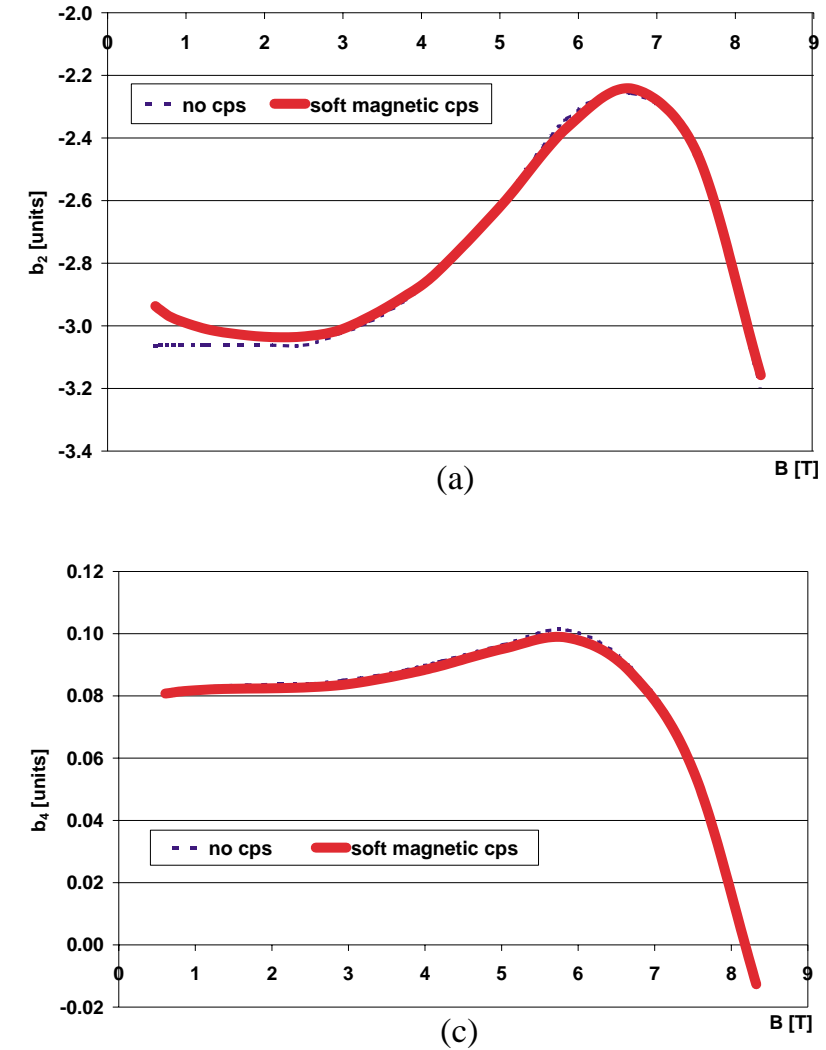

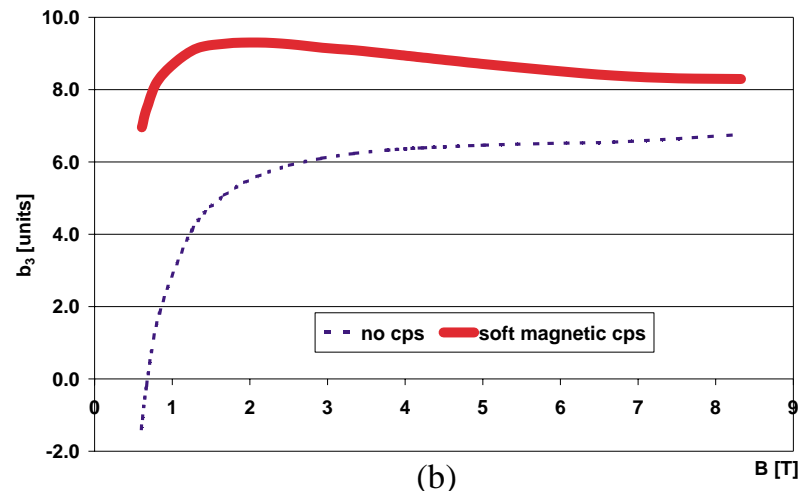

(b)

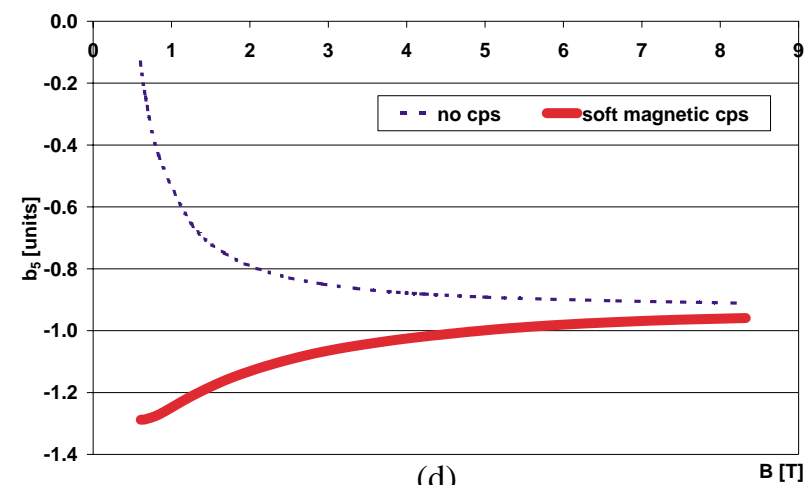

(d)

FIG. 9. (Color) Multipoles $b_{n}$ in units of $10^{-4}$ at $17 \mathrm{~mm}$ reference radius during current ramping (upramp LHC cycle); dashed lines: with nonmagnetic coil protection sheet (CPS); solid lines: with soft magnetic coil protection sheet. The soft magnetic coil protection sheet mainly influences the $b_{3}$ and $b_{5}$ component (reducing the nonlinearities in the upramp cycle on the multipole $b_{3}$ by a factor of 4 and of $b_{5}$ by a factor of 2 , respectively). The main objective in using the ferromagnetic coil protection sheet is the reduction of the multipole variation during the ramping of the magnets since any biasing can easily be reduced by coil block shiftings (see text for further details).

optimizing the contradictory objectives $\Delta b_{3}$ and $\Delta b_{5}$ with the ROXIE program. Table II shows the computed multipoles at different angles.

\section{CONCLUSION}

An elliptically layered current model for the filaments has been developed and is combined with the BEM-FEM method in order to compute persistent currents in superconducting magnet coils surrounded by an arbitrarily shaped iron yoke with highly nonlinear features. Because of the discretization in elliptic layers, the nonfully penetrated state of individual filaments could be taken into account as well as changes in current distribution arising from alternating external fields. The effect of a ferromagnetic coil protection sheet has been computed and optimized in order to act as an intrinsic corrector scheme to compensate for persistent current induced field errors. This sheet results in a reduction of the variation of all lower multipoles. 

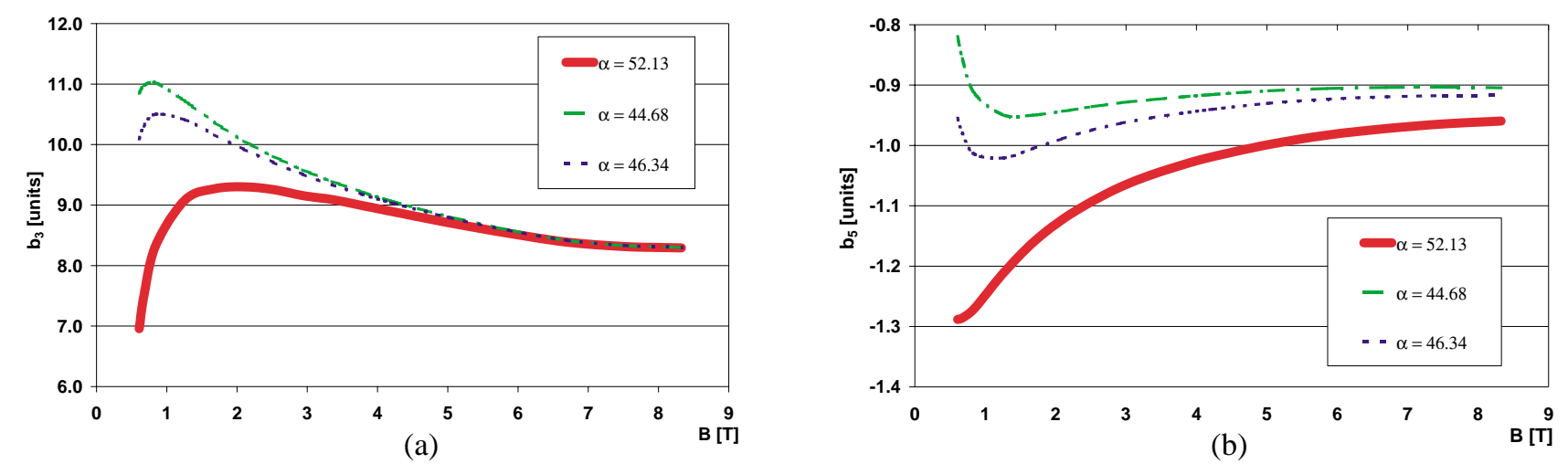

FIG. 10. (Color) Influence of angular variation of the coil protection sheet on the multipoles during ramping (upramp LHC cycle); top: $b_{3}$ for $\alpha=52.13^{\circ}$ (fully covered coil), $44.68^{\circ}$, and $46.34^{\circ}$; bottom: $b_{5}$ for $\alpha=52.13^{\circ}$ (fully covered coil), $44.68^{\circ}$, and $46.34^{\circ}$. The angle $\alpha$ is the covering angle relative to the abscissa (see Fig. 6). The coil protection sheet is assumed to be made of soft magnetic iron sheets $(99.99 \%$ pure $\mathrm{Fe})$. Multipoles are in units of $10^{-4}$ at $17 \mathrm{~mm}$ reference radius.

TABLE II. Expected relative multipole errors including persistent current contribution in units of $10^{-4}$ at 17 mm reference radius for different angles of the soft magnetic coil protection sheet.

\begin{tabular}{|c|c|c|c|c|c|c|}
\hline & Injection field & $\begin{array}{c}\alpha=44.68^{\circ} \\
\text { Nominal field }\end{array}$ & Variation $\Delta b_{n}$ & Injection field & $\begin{array}{c}\alpha=46.34^{\circ} \\
\text { Nominal field }\end{array}$ & Variation $\Delta b_{n}$ \\
\hline$b_{2}$ & -2.943 & -3.178 & 0.909 & -2.941 & -3.177 & 0.906 \\
\hline$b_{3}$ & 10.848 & 8.300 & 2.721 & 10.094 & 8.304 & 2.200 \\
\hline$b_{4}$ & 0.080 & -0.013 & 0.111 & 0.080 & -0.013 & 0.111 \\
\hline$b_{5}$ & -0.820 & -0.905 & 0.130 & -0.955 & -0.917 & 0.104 \\
\hline$b_{6}$ & 0.002 & -0.002 & 0.005 & 0.002 & -0.002 & 0.005 \\
\hline$b_{7}$ & 0.171 & 0.632 & 0.461 & 0.183 & 0.632 & 0.449 \\
\hline
\end{tabular}

[1] The LHC Study Group, The Large Hadron Collider: Conceptual Design (CERN, Geneva, 1995).

[2] Charles P. Bean, Rev. Mod. Phys. 36, 31-39 (1964).

[3] Luca Bottura, in Proceedings of the 16th International Conference on Magnet Technology, Florida, 1999 (IEEE, New York, 2000).

[4] Stefan Kurz and Stephan Russenschuck, Electr. Eng. (Germany) 82, 1-10 (1999).

[5] Martin N. Wilson, Superconducting Magnets (Oxford University, New York, 1983).

[6] Stefan Kurz, Joachim Fetzer, and Günther Lehner, IEEE Trans. Magn. 33, 1772-1775 (1997).
[7] H. Brück, R. Meinke, F. Müller, and P. Schmüser, Z. Phys. C 44, 385-392 (1989).

[8] V. V. Kashikhin and A. V. Zlobin, Fermilab Internal Report No. TD-99-049, 1999.

[9] Michael A. Green, IEEE Trans. Magn. 24, 823-826 (1988).

[10] Michael A. Green, in Proceedings of the 3rd Annual International Industrial Symposium on the Supercollider, Atlanta, 1991 (Plenum, New York, 1991).

[11] Rob Wolf and Sandrine Le Naour, CERN, LHC Project Note No. 230, 2000. 